\title{
Dermatology Case Reports
}

\section{Pemphigus Erythematosus in a Middle Aged Nepali Male: Case Report and Literature Review}

\section{Bibush Amatya $^{1 *}$, Aarif Syed MM¹ and Leison Maharjan²}

${ }^{1}$ Department of Dermatology and Venereology, Institute of Medicine, Tribhuvan University Teaching Hospital, Maharajgunj, Kathmandu, Nepal

${ }^{2}$ Department of Ear, Nose and Throat, Institute of Medicine, Tribhuvan University Teaching Hospital, Maharajgunj, Kathmandu, Nepal

\begin{abstract}
Introduction: Pemphigus erythematosus, also known as the Senear-Usher syndrome, is an autoimmune condition where there is overlap between the clinical and immunological features of pemphigus foliaceous and lupus erythematosus. Patients present with erythematous, scaly, and crusted lesions in seborrhoeic distribution along with malar rash in butterfly distribution. Diagnosis is based on clinical findings, characteristic histopathologic changes, presence of antinuclear antibodies and immunolabelling in direct immunofluorescence test.
\end{abstract}

Case Presentation: We report a case of a middle-aged Nepali male who presented with the complaints of photosensitivity, itchy rash on the cheeks and flaccid blisters and crusted erosions on the chest for a few weeks. His diagnosis of Senear-Usher syndrome was based on clinical, histological and immunological findings. He was subsequently started on oral prednisolone and hydroxychloroquine sulfate, along with topical corticosteroids, which induced remission within the first week. He is currently on maintenance therapy without any relapse. This is to our knowledge, the first case of Senear-Usher syndrome reported from Nepal.

Conclusion: Topical and oral corticosteroids along with hydroxychloroquine sulfate should be considered first line in the treatment of Senear-Usher syndrome.

Keywords: Pemphigus erythematosus; Senear-Usher syndrome; Immunological features

Abbreviations: ANA: Antinuclear Antibody; dsDNA: Double Stranded Deoxyribonucleic Acid; ESR: Erythrocyte Sedimentation Rate; IgG: Immunoglobulin G; C3: Complement 3, mL: Millilitre; mm: Millimetre; mm/Hg: Millimetre per 1 Column of Mercury

\section{Introduction}

Pemphigus erythematosus, also known as the Senear-Usher syndrome, is one of the variants of pemphigus, which is an autoimmune bullous disorder [1]. It is characterized by overlapping features of lupus erythematosus and pemphigus foliaceous. While the erythematous lesions on the face in a butterfly distribution resemble lupus erythematosus, the vesicles and crusted plaques on the trunk simulate pemphigus foliaceous [2]. Immunological features of both the diseases are also present. It is a rare condition, has been reported infrequently, with one review quoting a total of only 116 cases [1], and to the best our knowledge, has never been reported from Nepal. We report a case of a middle aged male presenting with features of pemphigus erythematosus with the relevant literature review.

\section{Case Presentation}

\section{Medical history}

A 42-year-old male presented with small fluid filled lesions on his scalp on and off for the last 7 months. The patient was apparently well 7 months back, when he developed red itchy lesions on the malar regions of the cheeks and the bridge of the nose in a butterfly distribution. This was accompanied by photosensitivity and increased loss of hair on the scalp but there were no oral or nasal ulcers, joint pain, chest pain and neurological symptoms. At the same time, he developed fluid filled lesions on the scalp, chest and upper back, which followed the previous course. The blisters were flaccid containing clear fluid, which ruptured within 1-2 days or immediately after manipulation. Rupture of the vesicles resulted in the formation of crusts, which healed with residual hyperpigmentation. Crops of new lesions occurred, which followed a similar course to leave hyperpigmentation. He visited a local clinic where he was given oral cloxacillin $500 \mathrm{mg}$ capsules to be taken four times a day for 10 days and topical combination of betamethasone valearate cream and neomycin cream to be applied on the affected sites for 2 weeks. After administering these medications as prescribed, his lesions healed in a month.

He was symptom free for the next 6 months following which there was severe relapse of the previous symptoms of fluid filled lesions on the scalp, chest and upper back, red rash on the face in a butterfly distribution, photosensitivity, malaise and diffuse loss of hair on the scalp. There was no history of prior admission to any hospital for medical or surgical reasons and no history of any chronic disease. There was no sudden gain or loss in weight and he was not on any medication prior to the eruption of the lesions.

\section{Clinical presentation}

When he presented to our department, we initially suspected a bullous disorder. His vitals were stable; there was no pallor, icterus, lymphadenopathy, cyanosis, clubbing, oedema or dehydration. No abnormalities were detected on systemic examination.

On dermatologic examination, he had Fitzpatrick skin type IV Flaccid vesicles and crusted erosions and plaques were present on the scalp, upper chest and back (Figure 1), malar rash in a butterfly

*Corresponding author: Bibush Amatya, Department of Dermatology and Venereology, Institute of Medicine, Tribhuvan University Teaching Hospital, Maharajgunj, Kathmandu, Nepal, Tel: +90-332-223-6204; E-mail: doc_bibush@hotmail.com

Received: August 09, 2017; Accepted: August 16, 2017; Published: August 23, 2017

Citation: Amatya B, Aarif Syed MM, Maharjan L (2017) Pemphigus Erythematosus in a Middle Aged Nepali Male: Case Report and Literature Review. Dermatol Case Rep 2: 125.

Copyright: (c) 2017 Amatya B, et al. This is an open-access article distributed unde the terms of the Creative Commons Attribution License, which permits unrestricted use, distribution, and reproduction in any medium, provided the original author and source are credited. 
distribution on the face and discoid rashes on the forehead (Figure 2). Nikolsky sign was positive while the Asboe-Hansen sign was negative. Oral and genital ulcers were absent.

\section{Laboratory tests}

His laboratory investigations revealed ANA positivity with dsDNA value of only $5 \mathrm{IU} / \mathrm{ml}$. There were no abnormalities in his haematological and biochemistry profiles and his routine urine microscopic examination did not reveal any anomalies except for a raised ESR of $45 \mathrm{~mm}$ in 1 hour.

\section{Histopathological and immunofluorescence studies}

Skin biopsies were taken from a fresh blister for histopathologic examination and from perilesional skin for direct immunofluorescence study. The histopathological examination revealed an acanthotic epidermis with large subcorneal blisters containing numerous neutrophils (Figure 3). Acantholytic cells were not detected. The

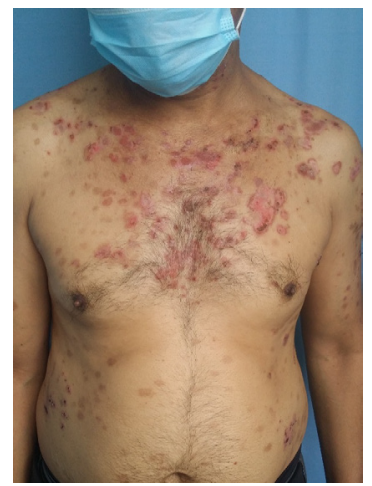

Figure 1: Erosions, crusted plaques and residual post-inflammatory hyperpigmentation on the chest and abdomen.

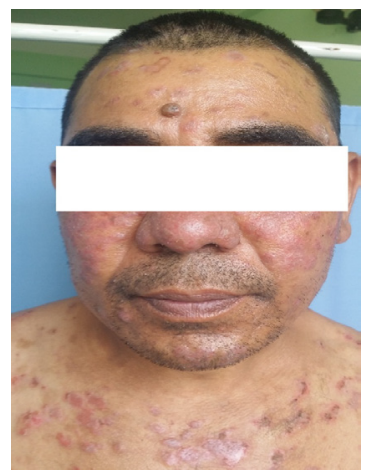

Figure 2: Malar rash and discoid rash on face.

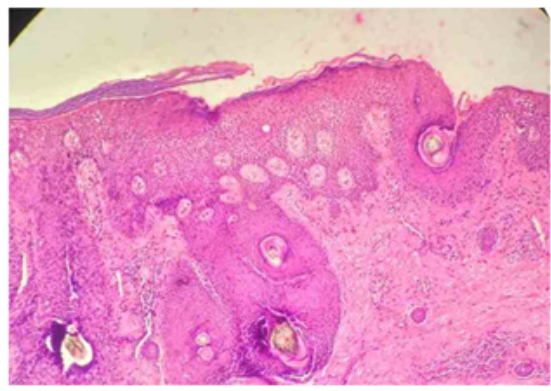

Figure 3: Histopathological examination revealing acanthotic epidermis with large subcorneal blisters.

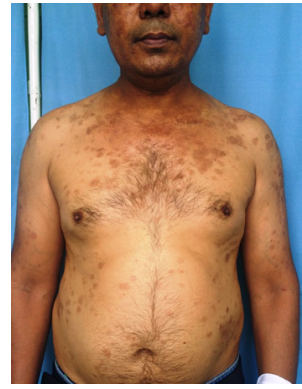

Figure 4: Residual hyperpigmentation on the chest.

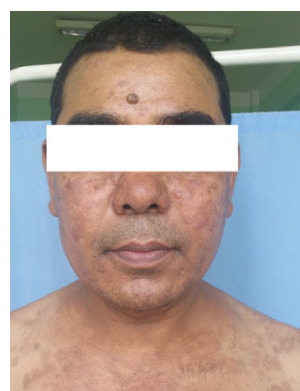

Figure 5: Resolution of malar rash.

dermis showed increase in number of capillaries and inflammatory cell infiltrates. The direct immunofluorescence revealed IgG 3+ and C3 1+ in intercellular space of epidermis and at the dermo-epidermal junction.

\section{Treatment}

Hewassubsequently diagnosed as suffering fromlupus erythematosus and was put on topical fluticasone propionate $0.05 \% \mathrm{w} / \mathrm{w}$, oral prednisolone at a dose of $1 \mathrm{mg} / \mathrm{kg}$ once daily, oral hydroxycholoroquine sulfate $200 \mathrm{mg}$ twice daily, a broad-spectrum sunscreen and prophylactic doses of rabeprazole, calcium and alendronate.

\section{Outcome}

He made a full recovery (Figures 4 and 5) within a month and has been maintained on hydroxychloroquine sulfate $100 \mathrm{mg}$ twice daily and tapering doses of oral prednisolone.

\section{Discussion}

Pemphigus erythematosus, is a rare condition that combines the clinical and immunological features of pemphigus foliaceous and lupus erythematosus [3]. Clinical features of the Senear Usher syndrome include the pemphigus foliaceous-like scattered scaly flaccid blisters with erosions and crusts on 'seborrhoeic' areas of the scalp, face, chest and upper back along with malar rash simulating lupus erythematosus [2] with absence of mucous membrane involvement [4]. The immunological features include IgG and C3 in the suprabasal layers as in pemphigus foliaceous and along the basement membrane zone similar to lupus erythematosus [5].

Many authors have disputed the existence of this syndrome as a distinct entity and consider it to be an abortive form of pemphigus foliaceous [6]. This could be supported by the study done by Oktarina et al. [7], where they demonstrated deposition of antibodies against desmoglein 1 on the basement membrane zone mimicking the lupus band test. This could explain the direct immunofluorescence findings when ANA reactivity is negative or weakly positive. 
Other authors have however stated that while pemphigus erythematosus has concomitant basement membrane zone deposition of immunoglobulin and complement in addition to intercellular epidermal staining, pemphigus foliaceous lacks basement membrane zone staining [8]. Studies have confirmed multiple-autoimmune process in this syndrome [7,9]. In fact, Chorzelski et al. [9] described the presence of immunoglobulins and complement at the basement membrane zone along with anti-epithelial antibodies in this syndrome in 1968. Then, 44 years later, Perez-Perez et. al. [5] not only confirmed the co-existence of antiepithelial and antinuclear antibodies but also ruled out cross-reactivity by immunochemical studies. These findings do support the hypothesis claiming the Senear-Usher syndrome to be a distinct entity and not just a variant of pemphigus foliaceous. Our patient satisfied both clinical and immunological features of the SenearUsher syndrome.

The differentials other than pemphigus erythematosus that can be considered are seborrhoeic dermatitis, bullous pemphigoid, and dermatitis herpetiformis. The characteristic appearance of seborrhoeic dermatitis is that of red, flaking, greasy scales on the scalp, nasolabial folds, ears, eyebrows and chest and not that of flaccid vesicles and bullae. The histopathology usually reveals 'squirting' of granulocytes from the dermal papilla. Oedema, hyperkeratosis and focal parakeratosis are also commonly seen. Bullous pemphigoid lesions are tense blisters occurring on normal or erythematous skin associated with severe pruritus. The blisters are typically located on flexor surfaces of the arms and legs, axillae, groin, and abdomen. Dermatitis herpetiformis has a chronic course, is characterized by pruritic papules and vesicles distributed on extensor surfaces, is associated with gluten sensitive enteropathy, demonstrates presence of neutrophils in dermis in histopathological sections, and IgA deposition on the papillary tips in direct immunofluorescence.

The course is often chronic lasting months to years. The treatment options for patients with pemphigus erythematosus include oral and topical corticosteroids, $\beta$-blockers, immunosuppressive agents, Jamarson therapy, antimalarials, sulfapyridine and anticholinergic agents [1]. The use of these medications can lead to partial or complete remission. However, they are associated with significant side effects and have to be monitored appropriately.

To the best of our knowledge, this is the first case report on this syndrome in Nepal. An extensive search of Pubmed, Ovid Medline, Ovid Embase and Google Scholar did not reveal any previously published case reports or case series from Nepal, although there have been case reports and case series published from the USA, India [6], Italy, Nigeria and Sudan.

\section{Conclusion}

Our patient was diagnosed with pemphigus erythematosus based on clinical and immunological findings. He responded promptly to topical and oral corticosteroids and hydroxychloroquine sulfate not necessitating the use of adjuvant immunosuppressants.

Topical and oral corticosteroids along with hydroxychloroquine sulfate should be considered first line in the treatment of such conditions.

\section{Declaration}

\section{Ethics approval and consent to participate}

Written informed consent was taken from the patient for collection of photographs, digital recording of clinical information and for dissemination of the information in a printed or digital form. All efforts have been made to ensure anonymity of the subject. The patient has been informed of their right to withdraw consent at any future point in time before online publication.

\section{Consent for publication}

Written informed consent was taken from the patient for publication of this case report. The patient has been informed of his right to withdraw consent at any future point in time before online publication.

\section{Availability of data and materials}

A copy of all the records of the patient including the photographs can be retrieved from the corresponding author.

\section{Competing interests}

The authors declare that they have no competing interests.

\section{Funding}

The authors have not received any funding for this research from any funding agency in the public, commercial, or not-for-profit sectors.

\section{Authors' Contributions}

BA was involved in writing the first drafts of case report and provided the photographs. SMMA was involved in critical revision of the manuscript and rewriting of the discussion and conclusion. LM searched all the relevant literature re-wrote the report, collected the informed consent from the participants and made revisions to the draft. All the authors gave their final approval for this version of the manuscript to be published.

\section{Acknowledgements}

The authors would like to thank the participant for granting their approval to write and publish this case report. The authors are also grateful to the faculty members and residents of the Department of Dermatology and Venereology at the Institute of Medicine, Tribhuvan University Teaching Hospital, Maharajgunj, Kathmandu, Nepal for their encouragement in preparing this case report.

\section{References}

1. Amerian ML, Ahmed AR (1985) Pemphigus erythematosus. Int J Dermatol 24:16-25.

2. Schmidt E, Groves R (2016) Immunobullous diseases. Rook's Textbook of Dermatology.

3. Steffen C, Thomas D (2003) The men behind the eponym. Senear FE, Usher B (eds), and the Senear-Usher syndrome. Am J Dermatopath 25:432-436.

4. Maize JC, Green D, Provost TT (1982) Pemphigus foliaceus: A case with serologic features of Senear-Usher syndrome and other autoimmune abnormalities. J Am Acad Dermatol 7: 736-741.

5. Pérez-Pérez ME, Avalos-Díaz E, Herrera-Esparza R (2012) Autoantibodies in senear-usher syndrome: Cross-reactivity or multiple autoimmunity? Autoimmune Diseases 1: 7

6. Chavan SA, Sharma YK, Deo K, Buch AC (2013) A case of senear-ushe syndrome. Indian J Dermatol 58:329.

7. Oktarina DA, Poot AM, Kramer D, Diercks GF, Jonkman MF, et al. (2012) The IgG "lupus-band" deposition pattern of pemphigus erythematosus: Association with the desmoglein 1 ectodomain as revealed by 3 cases. Arch Dermatol 148:1173-1178.

8. Hashimoto T (2003) Recent advances in the study of the pathophysiology of pemphigus. Arch Dermatol Res 295: S2-S11.

9. Chorzelski T, Jablonska S, Blaszczyk M (1968) Immunopathological investigations in the Senear-Usher syndrome (coexistence of pemphigus and lupus erythematosus). British J Dermatol 80: 211-217. 